\title{
Correction to: Analysis of Action Oriented Effects on Perceptual Process of Object Recognition Using Physiological Responses
}

\author{
Shanu Sharma, Anju Mishra, Sanjay Kumar, Priya Ranjan, \\ and Amit Ujlayan
}

\section{Correction to: \\ Chapter "Analysis of Action Oriented Effects on Perceptual Process of Object Recognition Using Physiological Responses" in: U. S. Tiwary (Ed.): Intelligent Human Computer Interaction, LNCS 11278, https://doi.org/10.1007/978-3-030-04021-5_5}

The original version of the chapter "Analysis of Action Oriented Effects on Perceptual Process of Object Recognition Using Physiological Responses", starting on p. 46 has been revised. The affiliations were mismatched to the author names in the XML version.

The original chapter was corrected.

\footnotetext{
The updated version of this chapter can be found at https://doi.org/10.1007/978-3-030-04021-5_5 